\title{
Reperfusion injury following cerebral ischemia: pathophysiology, MR imaging, and potential therapies
}

\author{
Jie Pan • Angelos-Aristeidis Konstas • Brian Bateman • \\ Girolamo A. Ortolano • John Pile-Spellman
}

Received: 16 March 2006 / Accepted: 24 October 2006 / Published online: 20 December 2006

(C) Springer-Verlag 2006

\begin{abstract}
Introduction Restoration of blood flow following ischemic stroke can be achieved by means of thrombolysis or mechanical recanalization. However, for some patients, reperfusion may exacerbate the injury initially caused by ischemia, producing a so-called "cerebral reperfusion injury". Multiple pathological processes are involved in this injury, including leukocyte infiltration, platelet and complement activation, postischemic hyperperfusion, and breakdown of the blood-brain barrier.

Methods/results and conclusions Magnetic resonance imaging (MRI) can provide extensive information on this process of injury, and may have a role in the future in stratifying patients' risk for reperfusion injury following recanalization. Moreover, different MRI modalities can be used to investigate the various mechanisms of reperfusion injury. Antileukocyte antibodies, brain cooling and conditioned blood reperfusion are potential therapeutic strategies for lessening or eliminating reperfusion injury, and interventionalists may play a role in the future in using some of these therapies in combination with thrombolysis or embolectomy. The present review summarizes the mechanisms of reperfusion injury and focuses on the way each of those mechanisms can be evaluated by different MRI modalities. The potential therapeutic strategies are also discussed.
\end{abstract}

J. Pan · A.-A. Konstas $(\bowtie) \cdot$ B. Bateman · J. Pile-Spellman

Department of Radiology, Columbia University,

177 Fort Washington Ave, MHB 8SK,

New York, NY 10032, USA

e-mail: ak2669@columbia.edu

G. A. Ortolano

Pall Corporation,

East Hills, NY, USA
Keywords Magnetic resonance imaging $\cdot$ Cerebral ischemia $\cdot$ Reperfusion injury $\cdot$ Hypothermia .

Conditioned blood reperfusion

\section{Introduction}

In the treatment of acute stroke, restoration of the blood supply can reduce more extensive brain tissue injured by salvaging a reversibly damage penumbra of tissue [1]. This mechanism provides a rationale for clinical trials which have demonstrated that reperfusion after thrombolysis improves clinical outcome in selected patients with acute stroke [2].

Reperfusion, however, carries certain risks. Some patients experience disastrous outcomes in the form of fatal edema or intracranial hemorrhage following thrombolysis [3]. In some animal stroke models $[4,5]$, reperfusion after a long ischemic period can cause a larger infarct than that associated with permanent vessel occlusion. Thus, while reperfusion may reduce infarct size and improve clinical outcome in some patients, in others it may exacerbate the brain injury and produce a so-called "cerebral reperfusion injury" $[4,6,7]$. Cerebral reperfusion injury can be defined as a deterioration of ischemic but salvageable brain tissue after reperfusion.

Thrombolysis [2] and embolectomy [8, 9] usually result in reperfusion of the infarcted brain tissue and therefore carry the risk of causing reperfusion injury. Thus reperfusion injury deserves the attention of those interested in the diagnosis and treatment of acute stroke. Strategies to reduce or minimize cerebral reperfusion injury require the understanding of the pathophysiology of cerebral reperfusion injury, and the way the reperfusion injury is visualized by magnetic resonance imaging (MRI). Therapeutic options 
for preventing or attenuating cerebral reperfusion need to be considered.

\section{Cerebral reperfusion injury: mechanisms and correlation with MRI}

Leukocyte infiltration and secondary ischemia as depicted with DW MRI

Leukocytes play an important role in the development of cerebral reperfusion injury. During reperfusion, activated leukocytes interact with endothelial cells and plug capillaries, disrupt the blood-brain barrier (BBB) through the release of neutrophil-derived oxidants and proteolytic enzymes, extravasate from capillaries and infiltrate brain tissue, and release cytokines which mediate inflammation. These processes produce an inflammatory cascade, resulting in the deterioration of the salvageable penumbra [7].

Evidence of the deleterious effects caused by leukocytes is provided by animal studies. For example, Zhang et al. [10], using a rat model of 2 hours transient focal cerebral ischemia induced by advancing a nylon monofilament to occlude the middle cerebral artery (MCA), showed that neutrophils accumulate at the site of neuronal injury 6 hours after restoration of cerebral circulation. The neutrophil accumulation occurred earlier and to a greater extent in reperfusion tissue than in tissue permanently deprived of blood supply [10]. Furthermore, this study showed that the infarct volume increased dramatically between 6 and 24 hours following the start of reperfusion, and that the period of maximal infarct expansion correlated closely with the time course of neutrophil infiltration.

The contribution of leukocytes to cerebral reperfusion injury is also supported by the beneficial effects of neutrophil depletion. Bednar et al. [11] investigated the effect of administering antineutrophil antiserum treatment on brain infarct size in a rabbit model of transient ischemia. The regional cerebral blood flow $(\mathrm{CBF})$ of neutropenic rabbits recovered from less than $5 \mathrm{ml} / 100 \mathrm{~g}$ per minute to 20-30 ml/100 g per minute following reperfusion, while in the non-neutropenic rabbits it remained at less than $10 \mathrm{ml} /$ $100 \mathrm{~g}$ per minute. Correspondingly, the infarct size was significantly smaller in the neutropenic animals. Similar results have been reported in rats treated with antineutrophil monoclonal antibodies [12].

The evidence from animal experiments points to the role of neutrophils in restricting $\mathrm{CBF}$ and increasing infarct size during reperfusion. High-quality evidence of reperfusioninduced secondary ischemic injury has been obtained using diffusion-weighted imaging (DWI) in animals. Furthermore, the evolution of reperfusion injury can be depicted by observing dynamic changes with DWI (Fig. 1) [13].
Olah et al. [13] investigated the dynamics of the apparent diffusion coefficient (ADC) in a rat model with 1 hour of MCA occlusion (MCAO) followed by 10 hours of reperfusion. They found that the ADC was decreased at the end of ischemia, then significantly improved during the first 2 hours of reperfusion (Fig. 2), but decreased again at later time points with a secondary increase of hemisphere lesion volume. The ADC change most likely reflects a secondary ischemic injury. Although a potential role for apoptosis cannot be excluded, the observed secondary deterioration of ADC did not support apoptosis as a major mechanism, because the ADC decline indicated cell swelling and not loss of cell volume, which is a fundamental and universal characteristic of programmed cell death [14]. Similarly, Neumann-Haefelin et al., also using a rat MCAO model, showed that DWI abnormalities reversed transiently during the early reperfusion period but recurred after several hours of restored blood flow [15]. Likewise, Li et al., using a rat model with 10-30 minutes of temporary MCAO, observed that the DWI hyperintensity seen during the initial ischemic insult reverted to normal approximately 60-90 minutes after the start of reperfusion, with secondary hyperintensities emerging 12 hours after reperfusion [16]. The secondary increase of DWI signal intensity observed in these studies is likely a marker of cerebral reperfusion injury [15].

Several interesting characteristics of the ADC changes during reperfusion have been reported in animal studies. First, the time of onset of the secondary reduction of ADC varies amongst different animal studies, ranging from 2 72 hours after reperfusion [12, 16-18]. The reason is probably due to the different durations of the initial ischemic insult and differences in the animal model used. Second, the secondary deterioration of ADC can be predicted by early changes of $\mathrm{T}_{2}$ during reperfusion. Olah et al. [13], in their rat model study, observed that an increase in $T_{2}$ value early in the ischemic period predicts a secondary deterioration in ADC after reperfusion. This suggests the possibility of using $\mathrm{T}_{2}$ value clinically to predict the extent of reperfusion injury likely to follow recanalization, and may be useful in assessing patients' likelihood of benefiting from thrombolysis.

Hemorrhagic transformation (HT) follows hyperperfusion and disruption of the BBB (see below), but some of the imaging studies are reviewed in this section because there is a close relationship between the ADC changes and the subsequent HT after cerebral ischemia. In a rabbit model of thromboembolic stroke, Adami et al. [19] compared the percentage of pixels with very low ADC at various time points after the start of ischemia with the subsequent occurrence of HT (as determined on histopathological sections). The authors found that, at each time point, regions populated by pixels with an ADC value 


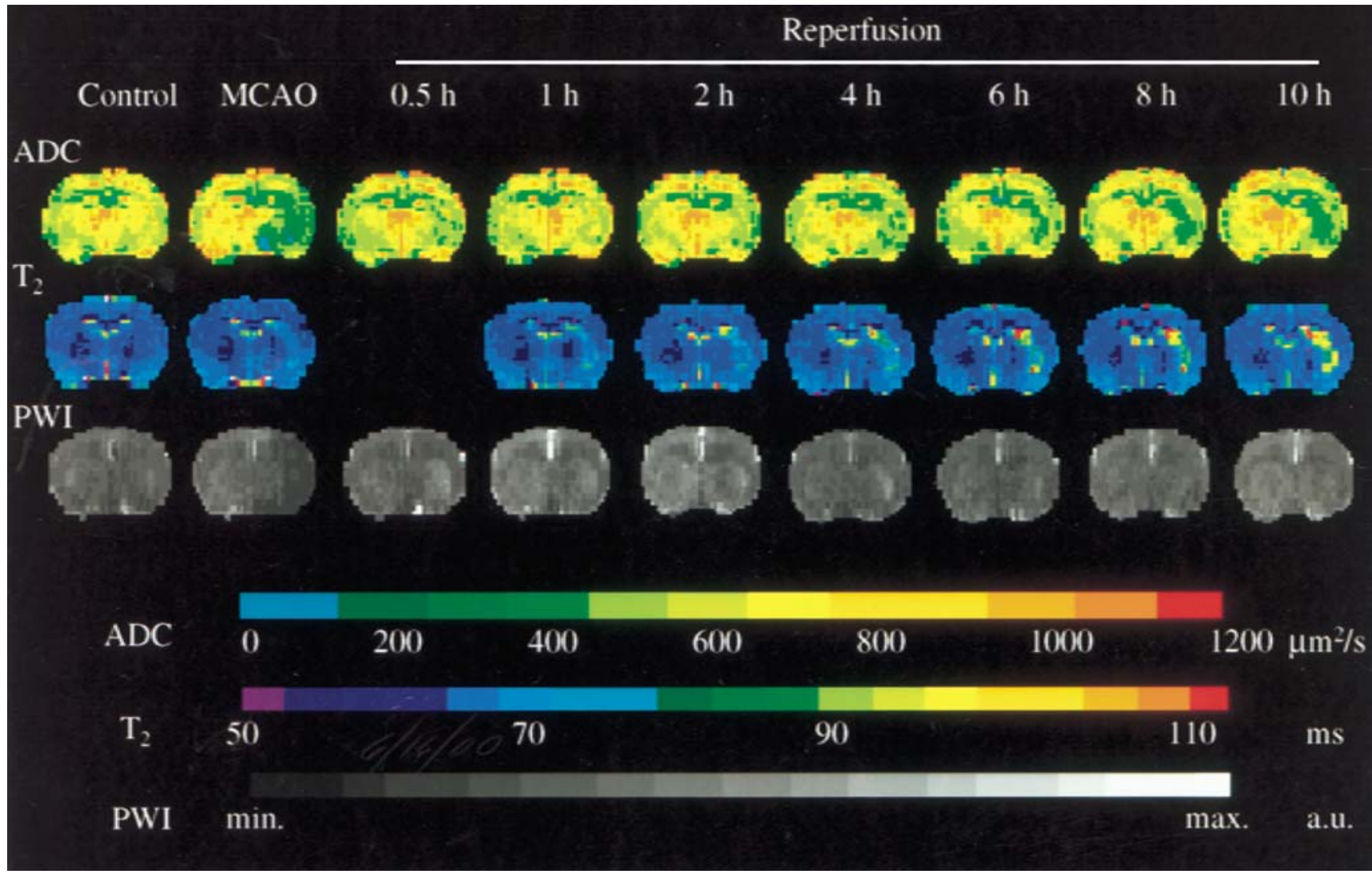

Fig. 1 DWI images of a rat model with 1 hour of MCA occlusion $(M C A O)$ followed by 10 hours of reperfusion. These images show the evolution of $\mathrm{ADC}$ at different times: before ischemia (control), at the end of MCAO, and at different time points of reperfusion. Note the transient recovery of ADC during the early phase of reperfusion, followed by secondary deterioration (reproduced with permission from Olah et al. [13])

of 80 or more, whereas eight of ten patients in the nonrecanalization group had a Barthel index of 50 or less.

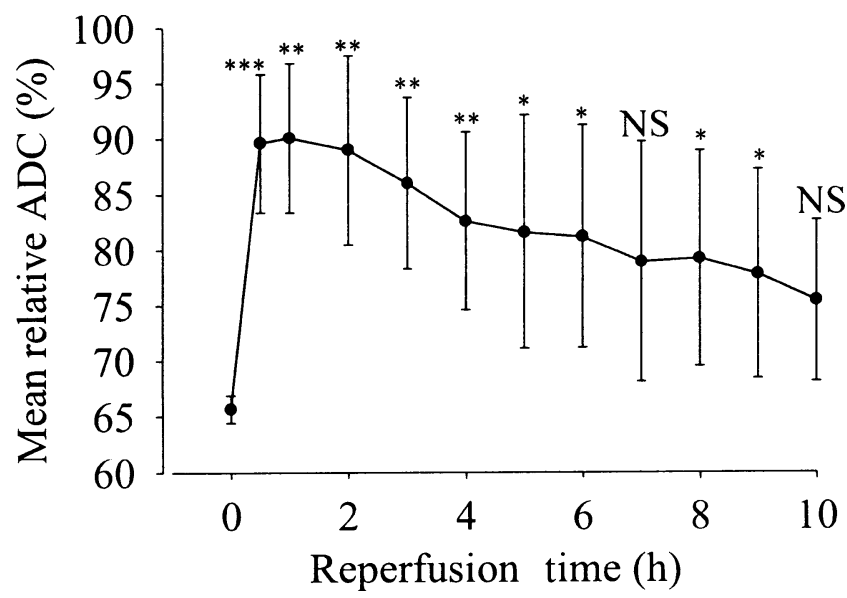

Fig. 2 Temporal evolution of mean relative ADC in the end-ischemic lesion volume in a rat model with 1 hour of MCA occlusion (MCAO) followed by 10 hours of reperfusion. Although a significant improvement in ADC attends reperfusion, there is a secondary deterioration likely as a consequence of the reperfusion-related injury (reproduced with permission from Olah et al. [13]) 
Molecular mechanisms of leukocyte infiltration

The mechanisms of leukocyte infiltration are characterized by leukocyte activation, leukocyte-endothelial interaction resulting in accumulation in the vascular bed, followed by leukocyte extravasation into the interstitial space. Each of these mechanisms is briefly reviewed.

The accumulation of leukocytes, along with red blood cells and platelets during reperfusion, may plug capillaries, preventing the restoration of blood flow and resulting in a "no-reflow" phenomenon and secondary cerebral ischemia [22-27]. Del Zoppo et al. [27] used a microscopic imaging system in a baboon model of transient ischemia. There was significant capillary obstruction caused by leukocytes reperfusing the microvascular bed within 60 minutes after the restoration of cerebral circulation. The "no-reflow" phenomenon explains well the better recovery of regional CBF in neutropenic rabbits [11].

The molecular mechanisms by which leukocytes infiltrate the parenchyma during reperfusion have been elucidated. This multistage process can be conceptually divided as follows: (1) leukocytes "rolling" on the endothelium, (2) firm adherence of leukocytes to the endothelium, and (3) transmigration into the tissue [1, 28-31]. Superoxide free radicals produced during ischemia and reperfusion cause the upregulation of endothelial P-selectin. Once leukocytes reach the ischemic territory the endothelial P-selectin interacts with its leukocyte counter-receptor P-selectin glycoprotein 1 [31]. This initial interaction facilitates the low affinity "rolling" of leukocytes on the endothelium. Firm adherence is developed with subsequent interaction of the leukocyte $\beta_{2}$ integrins CD11a/CD18 and CD11b/ CD18 with endothelial intercellular adhesion molecule 1 (ICAM-1).

The transmigration of leukocytes results from the expression of platelet-endothelial cell adhesion molecule-1 (PECAM-1) along the endothelial cell junction. Once the leukocytes infiltrate the parenchyma, they then release various chemical mediators including neutrophil elastase, reactive oxygen species (ROS), leukotrienes, and prostaglandins, resulting in increased microvascular permeability, edema, thrombosis and parenchymal cell death [1, 27, 32]. This process compounds the initial injury that occurred in these tissues during the hypoxic insult.

\section{Platelet-mediated reperfusion injury}

Platelets play a synergistic role with leukocytes in reperfusion injury. Several studies suggest that platelets are activated after cerebral ischemia and reperfusion [33-35]. Following activation, platelets may adhere to both leukocytes and cerebral microvascular endothelial cells, contributing to the "no-reflow" phenomenon and furthering tissue injury. Additionally, activated platelets release a variety of biochemical mediators of tissue injury.

Several studies have confirmed that platelet activation occurs in patients with stroke. Zeller et al. [36] used flow cytometric detection of platelet adhesion molecules as a marker of platelet activation, and reported a substantially greater activation in acute stroke patients than in normal controls. Similarly, van Kooten et al. [37], using urinary excretion of 11-dehydro-thromboxane-B2 as a marker of platelet activation, demonstrated a $347 \%$ increase in the marker excretion in ischemic stroke patients compared with matched controls.

Chong et al. [38] investigated changes in plateletleukocyte adhesion using a rat model of transient MCA ischemia. The authors reported that platelet-neutrophil adhesion was significantly increased from $16.6 \%$ in the control group to $39 \%$ in the reperfusion group $(P<0.01)$. Platelets in the stroke group expressed high levels of Pselectin. It was hypothesized that enhancement of plateletleukocyte adhesion may be mediated by increased Pselectin expression on the platelet membrane.

Studies on the role of platelets in coronary reperfusion of the heart suggest that, in addition to causing mechanical obstruction, platelets can cause temporary vasospasm by releasing serotonin, thromboxane $\mathrm{A} 2$, and free radicals. This can cause diminished reperfusion of the ischemic territory [33]. Further, activated platelets can release mediators that may cause chemotaxis and migration of leukocytes, thus exacerbating the inflammatory cascade [33]. These mechanisms may also prove to be important in cerebral reperfusion injury after ischemic stroke.

\section{Complement-mediated reperfusion injury}

Experimental studies have shown that complement activation is an important component of reperfusion injury in several organ systems. Complement may be activated during reperfusion through the antibody-dependent classical pathway, the alternative pathway, or the MBL/MASP (mannan-binding lectin/mannan-binding lectin-associated serine proteases) pathway $[39,40]$. Complement activation results in the formation of several inflammatory mediators, including anaphylatoxins C3a, C5a, and the distal complement component C5b-9 (membrane attack complex, MAC) [39]. C5a and the MAC are the best-characterized effectors of tissue damage within the complement system [41, 42]. C5a may stimulate leukocyte infiltration into the ischemia and reperfusion injury-affected tissue by its strong chemotaxis action. C5a may also further amplify the inflammatory response by inducing the release of several proinflammatory cytokines, including IL-1, IL-6, monocyte chemoattractant protein 1 (MCP-1) and tumor necrosis factor $\alpha$ $(\mathrm{TNF}-\alpha)$. MAC can cause cellular membrane injury directly 
and can increase cell membrane permeability. Furthermore, MAC plays an essential role in mediating the recruitment of leukocytes to the reperfused tissue via local induction of IL8. Several agents that inhibit all or part of the complement components such as cobra venom factor (CVF) [43], C5a monoclonal antibodies [44], and $\mathrm{Cl}$ esterase inhibitor [45], have been shown to significantly attenuate the degree of reperfusion injury in animal models, providing further evidence that complement plays an important role in reperfusion injury.

\section{Postischemic hyperperfusion and PWI}

Postischemic hyperperfusion is recognized as a frequent occurrence in stroke. Several animal studies suggest that the restoration of cerebral circulation consistently results in a hyperperfusion phase. Hyperperfusion may contribute to the development of reperfusion injury by causing brain edema or hemorrhage. Further, following postischemic hyperperfusion, a phase of secondary hypoperfusion can occur, which results in harmful effects on the reperfused tissue $[1,46,47]$.

Heiss et al. [46] observed the effects of hyperperfusion in cats using a model of temporary MCA occlusion of varying durations $(30,60$, and 120 minutes). In the 30minute group, only a transient reactive hyperperfusion (compared with preocclusion values) was found. All animals in this group survived during the observation period, and no cerebral injury was found on histological study. In the 60- and 120-minute groups, the degree of hyperperfusion was significant, reaching up to $300 \%$ of preocclusion values. Most importantly, a clear difference was found between surviving and dying cats. In dying cats, postischemic hyperperfusion was long and severe, large infarcts developed and intracranial pressure increased fatally. In surviving cats postischemic hyperperfusion was transient and less severe, infarcts were smaller and intracranial pressure was significantly lower. A report of a similar study design suggested that the degree of hyperperfusion correlates significantly with occurrence of intracerebral petechial hemorrhages in the damaged cortex [47].

Perfusion-weighted imaging (PWI) provides quantitative and qualitative maps of $\mathrm{CBF}$, cerebral blood volume (CBV), and mean transit time (MTT), allowing identification of regions of relative hypo- and hyperperfusion [48]. Kidwell et al. [48] used PW MRI to characterize hyperperfusion in 12 patients following intra-arterial thrombolysis. Hyperperfusion was visualized in 5 of 12 patients. On day $7,79 \%$ of voxels with hyperperfusion demonstrated infarction, whereas only 36\% not showing hyperperfusion demonstrated infarction. Despite the voxel-by-voxel association of hyperperfusion with infarction, there were no significant differences in the degree of clinical improve- ment in patients with regions of hyperperfusion versus those without. It has been reported that reduction in volume of hypoperfused brain on PWI is a marker of response to treatment and improved function in ischemic stroke patients [49]. Larger future studies are necessary to assess the extent to which both hyperperfusion and hypoperfusion may result in unfavorable clinical outcome.

\section{Breakdown of the BBB and contrast-enhanced MRI}

Breakdown of the BBB during cerebral reperfusion may lead to the development of vasogenic edema, HT and infarction, all contributing to cerebral reperfusion injury. This hypothesis has been supported by several clinical and animal studies [5, 50-52].

Sage and Duffy [50] investigated the increase in BBB permeability after transient global cerebral ischemia in a rat model. Permeability of the BBB was increased at 15 minutes after reperfusion of the brain, and cerebral edema occurred 15-30 minutes after reperfusion. Another study reported a disruption of the BBB after 3 hours of reperfusion, following a 3-hour temporary occlusion [5]. In contrast, the BBB remained intact after 6 hours of occlusion in a permanent occlusion group. Moreover, the temporary occlusion group had increased ipsilateral hemispheric water content, suggesting that cerebral edema had occurred. In addition to these reports, numerous histological studies with various tracers have established that reperfusion may exacerbate vasogenic edema due to BBB disruption [6, 53, 54].

T2-weighted imaging is useful for detecting vasogenic edema. Neumann-Haefelin et al. [15] used several MRI techniques to monitor the dynamics of secondary ischemic damage, BBB disturbances, and development of vasogenic edema during the reperfusion phase after focal cerebral ischemia in rats. After 2.5 hours of ischemia, signal intensity on both DWI and T2-weighted images increased rapidly in the previously ischemic region due to $\mathrm{BBB}$ disruption (enhancement on postcontrast T1-weighted images) and edema formation. More importantly, early BBB damage after reperfusion appeared to be predictive of relatively pronounced increases in $\mathrm{T} 2$ lesion size due to vasogenic edema at subacute time points (1 to 2 days).

Using a novel MRI marker that can image BBB disruption, Latour et al. [55] studied a total of 144 acute stroke patients, to test the association between reperfusion, HT and clinical outcome. Nearly two-thirds (63\%) of the patients had evidence of reperfusion within 1 week. BBB disruption was more common in patients who reperfused $(45 \%)$ than in patients who did not reperfuse $(18 \%)$. Both HT and BBB disruption were more common in patients undergoing intra-arterial rtPA therapy ( $31 \%$ and $55 \%)$, than those not treated (14\% and $25 \%)$. In the reperfused group, 
patients with BBB disruption were more likely to have a poor clinical outcome $(63 \%)$ than those without disruption (25\%). Moreover, early BBB disruption was an independent predictor of HT. This was a key study because it associated early BBB disruption with HT and poor clinical outcome in humans.

Contrast-enhanced T1-weighted MRI can detect disruption of the BBB during reperfusion. Under normal physiological conditions, contrast agent does not cross the intact BBB. Mechanisms that cause BBB disturbances, such as reperfusion injury, result in leakage of the contrast agent into the brain. In a recent study, rats were subjected either to permanent MCA occlusion, reperfusion after 1 hour (early reperfusion), or reperfusion after 3 hours of MCAO (late reperfusion) [56]. This protocol allowed monitoring the evolution of BBB disruption during permanent or temporary MCAO. Contrast agent extravasation was found in all animals with permanent MCAO and latereperfusion animals, but in only $40 \%$ of early-reperfusion animals. Postischemic hyperperfusion was demonstrated in late-reperfusion animals, but not in early-reperfusion animals. Thus, using contrast-enhanced MRI, the disruption of the BBB was correlated with the hemodynamic and biophysical consequences of reperfusion.

Fenstermacher et al. [57] employed the Patlak plot processing of gadolinium-shifted T1 relaxation-time images to obtain quantitative estimations of BBB opening in rats. Following 2.5 hours of reperfusion, the "blood-tissue distribution" of gadolinium-T1-shifted protons was serially imaged every 3-4 minutes for 20-30 minutes. After that, the value of blood-to-brain transfer constants (K1) for gadolinium was calculated from the serial changes of the relaxation time via Patlak plots [58] of MRI-T1 datasets. The K1 for gadolinium in ischemia and reperfusion areas was around 200 times larger than that of normal areas, indicating a huge impairment of BBB function during ischemia and reperfusion. This approach may be utilized to monitor the time-course of $\mathrm{BBB}$ function in transient cerebral ischemia.

The disruption of the BBB has also been observed in ischemic stroke patients undergoing rtPA therapy. A retrospective study of 213 patients used the postcontrast enhancement of the cerebrospinal fluid (CSF) in FLAIR images (termed hyperintense acute reperfusion marker, HARM) to characterize early BBB disruption [59]. BBB disruption was more common in patients with reperfusion $(45 \%)$ than in patients without reperfusion $(18 \%, P=$ 0.006). In multiple logistic regression, reperfusion was the strongest independent predictor of early BBB disruption (OR $4.09,95 \%$ CI 1.28 to $13.1, P=0.018$ ). HARM was associated with HT and worse clinical outcome. This is a key study for two reasons: first, the association between reperfusion, BBB disruption and HT was established in humans, and second, it may be possible to reduce the complications of rtPA by targeting adjunctive therapies against BBB disruption. In another recent study, three of the six patients receiving rtPA showed early parenchymal enhancement, and all three of them went on to develop HT, whereas none of the patients without early parenchymal enhancement developed hemorrhage [60]. The results suggest that early parenchymal enhancement may be significantly correlated with subsequent symptomatic HT and may be helpful in identifying patients at risk after thrombolytic therapy.

\section{Potential therapeutic strategies}

With the progress made in the understanding of the mechanisms in cerebral ischemia and reperfusion injury, an increasing number of strategies have been developed for limiting or preventing further brain damage during reperfusion [61-64]. Many of these strategies interfere with leukocyte infiltration. Two previously described animal studies indicated that leukocyte depletion with either antineutrophil antiserum treatment or with antineutrophil monoclonal antibody leads to better recovery of regional $\mathrm{CBF}$ and significantly reduces infarct size after cerebral reperfusion [11, 12]. Similarly, anti-ICAM-1 monoclonal antibodies reduce neutrophil infiltration and lesion size after reperfusion in rats, while they are ineffective in permanent ischemia [65], suggesting that ICAM-1 may be an attractive therapeutic target that specifically addresses the problem of reperfusion injury.

However, the translation of these promising antileukocyte strategies into effective therapies in humans has been disappointing. A randomized controlled trial (RCT) evaluated the use of enlimomab, a murine ICAM-1 antibody, in 625 ischemic stroke patients [66]. Patients were randomized to receive either enlimomab for 5 days or placebo within 6 hours after stroke onset. At 90 days, the modified Rankin scale score was worse in patients treated with enlimomab. Moreover, the hazard of death was $43 \%$ higher on enlimomab than on placebo. There are several possible explanations for the failure of enlimomab to produce the desired effects. It is possible that the positive results seen with anti-ICAM therapy in animal studies do not reflect the immunological response in human stroke. Alternatively, ICAM-1 therapy may be effective only after reperfusion [65], and it is believed that only a minority of patients experience complete reperfusion after ischemic stroke [67]. Thus, it may have been more appropriate to try enlimomab in patients who received thrombolytic therapy.

Another RCT randomized 966 ischemic stroke patients presenting within 6 hours of acute stroke to either placebo or UK-279,276, a CD11b/CD18 inhibitor. Of these patients, 
204 also received tPA when indicated [68]. The trial was prematurely terminated when an interim analysis showed that the trial would be unable to demonstrate the benefit of this therapy in terms of improvement in Scandinavian Stroke Scale score at 90 days.

Some conventional drugs have been shown in animal experiments to provide effective neuroprotection. For example, studies in a rat model have demonstrated that both dextran sulfate and unfractionated heparin can effectively prevent leukocyte infiltration and reduce infarct volume after reperfusion [69, 70]. Although the actual mechanism is still unclear, these findings suggest a possible role for these agents alongside other therapeutic modalities that cause recanalization (i.e. intravenous/intra-arterial rtPA, mechanical thrombolectomy) [69, 70]. Trials investigating this approach in humans have yet to be conducted. Besides these drug therapies, there are two management strategies, brain cooling and conditioned blood perfusion, which are reviewed in some detail because they can modulate several pathways/mechanisms that contribute to reperfusion injury. Both strategies are promising, and interventional neuroradiologists may have a role in administering these therapies in the future.

\section{Brain cooling}

Reports on the role of hypothermia in humans are encouraging. Two large RCTs established conclusively the neuroprotective effects and safety of systemic hypothermia after cardiac arrest [71, 72]. A meta-analysis of the results [73] showed that patients in the hypothermia group were more likely to be discharged with no or only minimal neurological damage (risk ratio $1.68 ; 95 \%$ CI $1.29-2.07$ ). This translated to six as the a number needed to treat in order to prevent one death or serious neurological deficit.

There are no hypothermia efficacy studies in ischemic stroke patients. Several animal studies of temporary MCAO have shown that systemic hypothermia applied up to 3 hours after reperfusion has started is effective in reducing infarct volume (by $50-90 \%$ ) and reducing animal mortality [74, 75]. Moreover, hypothermia exerts its neuroprotective effects partly by suppressing the BBB opening [76, 77] and by attenuating neutrophil infiltration in the ischemic penumbra [78, 79]. Hence, hypothermia exerts at least some of its neuroprotective effects by modulating mechanisms responsible for reperfusion injury.

In most clinical studies, hypothermia is induced by surface cooling with the use of cooling blankets, alcohol applied to exposed skin, or ice bags to groin, axilla and neck. Although whole-body surface cooling is the simplest and most cost-effective option for inducing hypothermia [80], it has two major drawbacks. First, it takes several hours to reach the target body core temperature. All studies report a 3- to 7-hour time period for cooling down to 32 $34^{\circ} \mathrm{C}$ [81-84]. Target temperature should be reached as soon as possible to prevent reperfusion injury; the 3-7 hour time period to reach target temperature is too long and the therapeutic window will be missed for many patients. Theoretical models predict that intracerebral hypothermia can be achieved up to 30 times faster than whole-body hypothermia [85]. The second drawback of whole-body cooling is the high incidence of complications such as pneumonia, arrhythmias/bradycardias, hypotension and coagulopathies [81-83, 86, 87]. Selective brain cooling may dramatically improve the safety profile of therapeutic hypothermia. Selective brain cooling can be achieved by means of an endovascular catheter that infuses the cerebral vasculature with cold saline. A recent study examined whether cold saline $\left(20^{\circ} \mathrm{C}\right)$ infused intra-arterially into the ischemic territory of rats that had undergone MCA occlusion could selectively induce cerebral hypothermia and reduce brain injury from stroke [88]. Following cold saline infusion, cerebral temperature fell within $1 \mathrm{~min}$ to $33.4^{\circ} \mathrm{C}$, while rectal temperature was maintained above $36^{\circ} \mathrm{C}$ at all times. A significantly reduced infarct volume was found in ischemic rats that received a local cooling infusion compared with control animals (Fig. 3). Further clinical research is needed to explore the therapeutic potential of selective brain cooling and the yet-unknown adverse effects, as well as to establish the extent to which selective brain cooling specifically modulates reperfusion injury.

\section{Conditioned blood reperfusion}

Conditioned blood reperfusion is a therapeutic strategy in which the ischemic organ is perfused with autologous

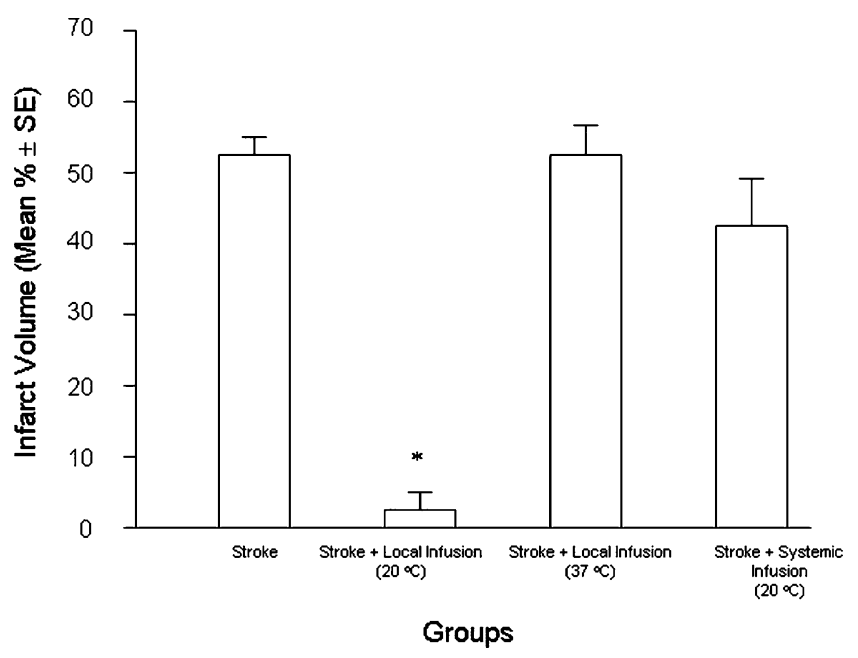

Fig. 3 Graph showing percentages of infarct volume in the four ischemic rat groups (stroke, stroke with local infusion of saline at $20^{\circ} \mathrm{C}$ and $37^{\circ} \mathrm{C}$, and stroke with systemic infusion at $20^{\circ} \mathrm{C}$ ). A significantly $(P<0.001)$ reduced infarct volume was found in ischemic rats that received a local cooling infusion compared with the rats in the other three groups (reproduced with permission from Ding et al. [88]) 
conditioned blood at the onset of reperfusion. Blood conditioned by filtration is depleted of leukocytes and platelets, both at levels far greater than $99.99 \%$, and the complement cascade is modulated in such a way as to prevent the deposition of MAC onto ischemic tissue [89]. Filtering the reperfused blood in this manner may reduce further tissue damage after restoration of circulation [89, 90]. Blood can be filtered using the conditioned blood reperfusion application (CoBRA) filter (Pall B-1328 filter; Pall Corporation, East Hill, N.Y.) at the onset of reperfusion [89]. Withdrawn arterial blood can be filtered through the CoBRA filter and returned back to the cerebral circulation via an endovascular catheter. A small feasibility and safety clinical trial of blood filtering during primary percutaneous transluminal coronary angioplasty for acute myocardial infarction demonstrated that integration of the CoBRA filter into an active hemoperfusion system did not delay the procedure or cause any complications [89]. Furthermore, conditioned blood reperfusion resulted in an acute improvement in ventricular function. Animal models of hypothermic circulatory arrest have demonstrated improvement of neurological outcomes following conditioned blood reperfusion [91-93]. In one of these studies, pigs received blood filtered using the CoBRA filter [92]. Each pig received a common carotid artery filtered perfusion for 10 minutes. Pigs receiving conditioned perfusion had significantly better neurological scores than control pigs $(P<0.005)$. These findings suggest that multifocal intervention aimed at leukocytes, platelets and complement attenuation can dramatically improve neurological recovery from ischemic insult. Future studies will establish the efficacy of this strategy and the incidence of possible adverse effects, such as coagulapathies and infections.

Further studies are needed to evaluate the role of local hypothermia and conditioned blood perfusion in reducing postischemic reperfusion injury. It is possible, yet still speculative, that in the future interventional neuroradiologists may use these approaches following recanalization with thrombolytic agents or mechanical embolectomy to lessen reperfusion injury.

Conflict of interest statement Girolamo A Ortolano, $\mathrm{PhD}$, is Vice President for Scientific Affairs of Pall Corporation. John Pile-Spellman, $\mathrm{MD}$, has received grant support for research from Pall Corporation.

\section{References}

1. Schaller B, Graf R (2004) Cerebral ischemia and reperfusion: the pathophysiologic concept as a basis for clinical therapy. J Cereb Blood Flow Metab 24:351-371
2. The National Institute of Neurological Disorders and Stroke rt-PA Stroke Study Group (1995) Tissue plasminogen activator for acute ischemic stroke. N Engl J Med 333:1581-1587

3. The National Institute of Neurological Disorders and Stroke rt-PA Stroke Study Group (1997) Intracerebral hemorrhage after intravenous t-PA therapy for ischemic stroke. Stroke 28:2109 2118

4. Aronowski J, Strong R, Grotta JC (1997) Reperfusion injury: demonstration of brain damage produced by reperfusion after transient focal ischemia in rats. J Cereb Blood Flow Metab 17:1048-1056

5. Yang GY, Betz AL (1994) Reperfusion-induced injury to the blood-brain barrier after middle cerebral artery occlusion in rats. Stroke 25:1658-1665

6. Dietrich WD (1994) Morphological manifestations of reperfusion injury in brain. Ann N Y Acad Sci 723:15-24

7. Kuroda S, Siesjo BK (1997) Reperfusion damage following focal ischemia: pathophysiology and therapeutic windows. Clin Neurosci 4:199-212

8. Gobin YP, Starkman S, Duckwiler GR et al (2004) A phase 1 study of mechanical embolus removal in cerebral ischemia. Stroke $35: 2848-2854$

9. Martinez H, Zoarski GH, Obuchowski AM et al (2004) Mechanical thrombectomy of the internal carotid artery and middle cerebral arteries for acute stroke by using the retriever device. AJNR Am J Neuroradiol 25:1812-1815

10. Zhang RL, Chopp M, Chen H, Garcia JH (1994) Temporal profile of ischemic tissue damage, neutrophil response, and vascular plugging following permanent and transient $(2 \mathrm{H})$ middle cerebral artery occlusion in the rat. J Neurol Sci 125:3-10

11. Bednar MM, Raymond S, McAuliffe T et al (1991) The role of neutrophils and platelets in a rabbit model of thromboembolic stroke. Stroke 22:44-50

12. Matsuo Y, Onodera H, Shiga Y et al (1994) Correlation between myeloperoxidase-quantified neutrophil accumulation and ischemic brain injury in the rat. Effects of neutrophil depletion. Stroke 25:1469-1475

13. Olah L, Wecker S, Hoehn M (2000) Secondary deterioration of apparent diffusion coefficient after 1-hour transient focal cerebral ischemia in rats. $\mathrm{J}$ Cereb Blood Flow Metab 20:1474-1482

14. Bortner CD, Cidlowski JA (1998) A necessary role for cell shrinkage in apoptosis. Biochem Pharmacol 56:1549-1559

15. Neumann-Haefelin T, Kastrup A, de Crespigny A et al (2000) Serial MRI after transient focal cerebral ischemia in rats: dynamics of tissue injury, blood-brain barrier damage, and edema formation. Stroke 31:1965-1972

16. Li F, Liu KF, Silva MD et al (2000) Transient and permanent resolution of ischemic lesions on diffusion-weighted imaging after brief periods of focal ischemia in rats: correlation with histopathology. Stroke 31:946-954

17. Li F, Han SS, Tatlisumak T et al (1999) Reversal of acute apparent diffusion coefficient abnormalities and delayed neuronal death following transient focal cerebral ischemia in rats. Ann Neurol 46(3):333-342

18. Lee SK, Kim DI, Jeong EK et al (2002) Temporal changes in reversible cerebral ischemia on perfusion- and diffusion-weighted magnetic resonance imaging: the value of relative cerebral blood volume maps. Neuroradiology 44:103-108

19. Adami A, Thijs V, Tong DC, Beaulieu C, Moseley ME, Yenari MA (2002) Use of diffusion weighted MRI to predict the occurrence and severity of hemorrhagic transformation in a rabbit model of embolic stroke. Brain Res 944:32-39

20. Selim M, Fink JN, Kumar S, Caplan LR, Horkan C, Chen Y, Linfante I, Schlaug G (2002) Predictors of hemorrhagic transformation after intravenous recombinant tissue plasminogen activa- 
tor: prognostic value of the initial apparent diffusion coefficient and diffusion-weighted lesion volume. Stroke 33:2047-2052

21. Taleb M, Lovblad KO, El-Koussy M et al (2001) Reperfusion demonstrated by apparent diffusion coefficient mapping after local intra-arterial thrombolysis for ischaemic stroke. Neuroradiology 43:591-594

22. Hallenbeck J, Dutka AJ, Tanishima T et al (1986) Polymorphonuclear leukocyte accumulation in brain regions with low blood flow during the early post ischemic period. Stroke 17:246-253

23. Suval WD, Duran WN, Boric MP et al (1987) Microvascular transport and endothelial cell alterations preceding skeletal muscle damage in ischemia and reperfusion injury. Am J Surg 154:211215

24. Janoff A, Schaefer S, Scherer J et al (1965) Mediators in inflammation in leukocyte lysosomes. mechanism of action of lysosomal cationic protein upon vascular permeability in the rat. J Exp Med 122:841-851

25. Vasthare US et al (1987) Effect of fentanyl and nubain on regional cerebral blood flow. Fed Proc 46:356

26. del Zoppo GJ, Mabuchi T (2003) Cerebral microvessel responses to focal ischemia. J Cereb Blood Flow Metab 23:879-894

27. del Zoppo GJ, Schmid-Schonbein GW, Mori E et al (1991) Polymorphonuclear leukocytes occlude capillaries following middle cerebral artery occlusion and reperfusion in baboons. Stroke 22:1276-1283

28. Harlan JM (1985) Leukocyte-endothelial interactions. Blood 65:513-525

29. Lefer AM, Ma XL, Weyrich A et al (1993) Endothelial dysfunction and neutrophil adherence as critical events in the development of reperfusion injury. Agents Actions Suppl 41:127135

30. Nishigaya K, Yoshida Y, Sasuga M, Nukui H, Ooneda G (1991) Effect of recirculation on exacerbation of ischemic vascular lesions in rat brain. Stroke 22:635-642

31. Jean WC, Spellman SR, Nussbaum ES, Low WC (1998) Reperfusion injury after focal cerebral ischemia: the role of inflammation and the therapeutic horizon. Neurosurgery 43:1382-1396

32. Carden DL, Granger DN (2000) Pathophysiology of ischemia and reperfusion injury. J Pathol 190:255-266

33. Gawaz M (2004) Role of platelets in coronary thrombosis and reperfusion of ischemic myocardium. Cardiovasc Res 61:498-511

34. Ko W, Lang D, Hawes AS et al (1993) Platelet-activating factor antagonism attenuates platelet and neutrophil activation and reduces myocardial injury during coronary reperfusion. J Surg Res 55:504-515

35. Rinder CS, Gaal D, Student LA, Smith BR (1994) Plateletleukocyte activation and modulation of adhesion receptors in pediatric patients with congenital heart disease undergoing cardiopulmonary bypass. J Thorac Cardiovasc Surg 107:280-288

36. Zeller JA, Tschoepe D, Kessler C (1999) Circulating platelets show increased activation in patients with acute cerebral ischemia. Thromb Haemost 81:373-377

37. van Kooten F, Ciabattoni G, Koudstaal PJ, Dippel DW, Patrono C (1999) Increased platelet activation in the chronic phase after cerebral ischemia and intracerebral hemorrhage. Stroke 30:546549

38. Chong ZZ, Xu QP, Sun JN (2001) Effects and mechanism of triacetylshikimic acid on platelet adhesion to neutrophil induced by thrombin and reperfusion after focal cerebral ischemia in rats. Acta Pharmacol Sin 22:679-684

39. Collard CD, Lekowski R, Jordan JE et al (1999) Complement activation following oxidative stress. Mol Immunol 36:941-948

40. D'Ambrosio AL, Pinsky DJ, Connolly ES (2001) The role of the complement cascade in ischemia/reperfusion injury: implications for neuroprotection. Mol Med 7:367-382
41. Arumugam TV, Shiels IA, Woodruff TM et al (2004) The role of the complement system in ischemia-reperfusion injury. Shock 21:401-409

42. Hart ML, Walsh MC, Stahl GL et al (2004) Initiation of complement activation following oxidative stress. In vitro and in vivo observations. Mol Immunol 41:165-171

43. Meyer ZU, Link C, Jorns A, Nagel E, Kohl J (2001) Preconditioning with the prostacyclin analog epoprostenol and cobra venom factor prevents reperfusion injury and hyperacute rejection in discordant liver xenotransplantation. Xenotransplantation 8:4147

44. Fung M, Fure H, Sun W, Sun C, Shi NY et al (2003) Preneutralization of $\mathrm{C} 5 \mathrm{a}$-mediated effects by the monoclonal antibody 137-26 reacting with the $\mathrm{C} 5$ a moiety of native $\mathrm{C} 5 \mathrm{a}$ without preventing C5 cleavage. Clin Exp Immunol 133:160-169

45. Horstick G (2002) C1-esterase inhibitor in ischemia and reperfusion. Immunobiology 205:552-562

46. Heiss WD, Graf R, Lottgen J, Ohta K, Wagner R (1997) Repeat positron emission tomographic studies in transient middle cerebral artery occlusion in cats: residual perfusion and efficacy of postischemic reperfusion. J Cereb Blood Flow Metab 17:388-400

47. Tamura A, Asano T, Sano K (1980) Correlation between rCBF and histological changes following temporary middle cerebral artery occlusion. Stroke 11:487-493

48. Kidwell CS, Saver JL, Mattiello J et al (2001) Diffusion-perfusion MRI characterization of post-recanalization hyperperfusion in humans. Neurology 57:2015-2021

49. Hillis AE, Wityk RJ, Beauchamp NJ et al (2004) Perfusionweighted MRI as a marker of response to treatment in acute and subacute stroke. Neuroradiology 46:31-39

50. Sage JI, Duffy TE (1984) Early changes in blood brain barrier permeability to small molecules after transient cerebral ischemia. Stroke 15:46-50

51. del Zoppo GJ, von Kummer R, Hamann GF (1998) Ischaemic damage of brain microvessels: inherent risk for thrombolytic treatment in stroke. J Neurol Neurosurg Psychiatry 65:1-9

52. Dobbin J, Crockard HA, Ross-Russell R (1989) Transient bloodbrain barrier permeability following profound temporary global ischemia: an experimental study using 14C-AIB. J Cereb Blood Flow Metab 9:71-78

53. Kuroiwa T, Shibutani M, Tajima T et al (1990) Vasogenic component of ischemic brain edema in experimental focal ischemia. Adv Neurol 52:548

54. Belayev L, Busto R, Zhao W et al (1996) Quantitative evaluation of blood-brain barrier permeability following middle cerebral artery occlusion in rats. Brain Res 739:88-96

55. Latour LL, Ezzeddine MA, Chalela JA, Warach S (2004) Early blood-brain barrier disruption in human focal brain ischemia. Ann Neurol 56:468-477

56. Kastrup A, Engelhorn T, Beaulieu C et al (1999) Dynamics of cerebral injury, perfusion, and blood-brain barrier changes after temporary and permanent middle cerebral artery occlusion in the rat. J Neurol Sci 166:91-99

57. Fenstermacher JD, Ewing JR, Nagaraja T, Nagesh V, Yee JS et al (2003) Estimating blood-brain barrier opening in a rat model of hemorrhagic transformation with Patlak plots of GdDTPA contrast-enhanced MRI. Acta Neurochir Suppl 86:3537

58. Patlak CS, Blasberg RG, Fenstermacher JD (1983) Graphical evaluation of blood-to-brain transfer constants from multiple-time uptake data. J Cereb Blood Flow Metab 3:1-7

59. Warach S, Latour LL (2004) Evidence of reperfusion injury, exacerbated by thrombolytic therapy, in human focal brain ischemia using a novel imaging marker of early blood-brain barrier disruption. Stroke 35[11 Suppl 1]:2659-2661 
60. Vo KD, Lin W, Hsu CY, Lee Y, Lee JM (2003) MR imaging enhancement patterns as predictors of hemorrhagic transformation in acute ischemic stroke. AJNR Am J Neuroradiol 24:674-679

61. van Bruggen N, Thibodeaux H, Palmer JT et al (1999) VEGF antagonism reduces edema formation and tissue damage after ischemia/reperfusion injury in the mouse brain. J Clin Invest 104:1613-1620

62. Li PA, He QP, Nakamura L et al (2001) Free radical spin trap alpha-phenyl-N-tert-butyl-nitron inhibits caspase-3 activation and reduces brain damage following a severe forebrain ischemic injury. Free Radic Biol Med 31:1191-1197

63. Rimpilainen J, Romsi P, Pokela M et al (2002) Lamotrigine plus leukocyte filtration as a neuroprotective strategy in experimental hypothermic circulatory arrest. Ann Thorac Surg 73:163-172

64. Wang GJ, Deng HY, Maier CM et al (2002) Mild hypothermia reduces ICAM-1 expression, neutrophil infiltration and microglia/monocyte accumulation following experimental stroke. Neuroscience 114:1081-1090

65. Zhang RL, Jiang N, Tang WX, Prostak J, Manning AM et al (1995) Anti-intercellular adhesion molecule-1 antibody reduces ischemic cell damage after transient but not permanent middle cerebral artery occlusion in the Wistar rat. Stroke 26:1438-1443

66. Enlimomab Acute Stroke Trial Investigators (2001) Use of antiICAM-1 therapy in ischemic stroke: results of the Enlimomab Acute Stroke Trial. Neurology 57:1428-1434

67. Zanette EM, Roberti C, Mancini G et al (1995) Spontaneous middle cerebral artery reperfusion in ischemic stroke. A follow-up study with transcranial Doppler. Stroke 26:430-433

68. Krams M, Lees KR, Hacke W et al (2003) Acute Stroke Therapy by Inhibition of Neutrophils (ASTIN): an adaptive dose-response study of UK-279,276 in acute ischemic stroke. Stroke 34:2543-2548

69. Yanaka K, Spellman SR, McCarthy JB et al (1996) Reduction of brain injury using heparin to inhibit leukocyte accumulation in a rat model of transient focal cerebral ischemia. I. Protective mechanism. J Neurosurg 85:1102-1107

70. Yanaka K, Spellman SR, McCarthy JB et al (1996) Reduction of brain injury using heparin to inhibit leukocyte accumulation in a rat model of transient focal cerebral ischemia. II. Dose-response effect and the therapeutic window. J Neurosurg 85:1108-1112

71. Bernard SA, Gray TW, Buist MD et al (2002) Treatment of comatose survivors of out-of-hospital cardiac arrest with induced hypothermia. N Engl J Med 346:557-563

72. Hypothermia after Cardiac Arrest Study Group (2002) Mild therapeutic hypothermia to improve the neurologic outcome after cardiac arrest. N Engl J Med 346:549-556

73. Holzer M, Bernard SA, Hachimi-Idrissi S et al (2005) Hypothermia for neuroprotection after cardiac arrest: systematic review and individual patient data meta-analysis. Crit Care Med 33:414-418

74. Huh PW, Belayev L, Zhao W et al (2000) Comparative neuroprotective efficacy of prolonged moderate intraischemic and postischemic hypothermia in focal cerebral ischemia. J Neurosurg 92:91-99

75. Maier CM, Sun GH, Kunis D et al (2001) Delayed induction and long-term effects of mild hypothermia in a focal model of transient cerebral ischemia: neurological outcome and infarct size. J Neurosurg 94:90-96

76. Huang F, Zhou L (1998) Effect of mild hypothermia on the changes of cerebral blood flow, brain blood barrier and neuronal injuries following reperfusion of focal cerebral ischemia in rats. Chin Med J (Engl) 111(4):368-372
77. Huang ZG, Xue D, Preston E et al (1999) Biphasic opening of the blood-brain barrier following transient focal ischemia: effects of hypothermia. Can J Neurol Sci 26:298-304

78. Ishikawa M, Sekizuka E, Sato S et al (1999) Effects of moderate hypothermia on leukocyte-endothelium interaction in the rat pial microvasculature after transient middle cerebral artery occlusion. Stroke 30:1679-1686

79. Toyoda T, Suzuki S, Kassell NF et al (1996) Intraischemic hypothermia attenuates neutrophil infiltration in the rat neocortex after focal ischemia-reperfusion injury. Neurosurgery 39:12001205

80. Feigin V, Anderson N, Gunn A et al (2003) The emerging role of therapeutic hypothermia in acute stroke. Lancet Neurol 2:529

81. Kammersgaard LP, Rasmussen BH, Jorgensen HS et al (2000) Feasibility and safety of inducing modest hypothermia in awake patients with acute stroke through surface cooling: a case-control study: the Copenhagen Stroke Study. Stroke 31:2251-2256

82. Krieger DW, De Georgia MA, Abou-Chebl A et al (2001) Cooling for acute ischemic brain damage (cool aid): an open pilot study of induced hypothermia in acute ischemic stroke. Stroke 32:1847-1854

83. Schwab S, Georgiadis D, Berrouschot J et al (2001) Feasibility and safety of moderate hypothermia after massive hemispheric infarction. Stroke 32:2033-2035

84. Steiner T, Friede T, Aschoff A et al (2001) Effect and feasibility of controlled rewarming after moderate hypothermia in stroke patients with malignant infarction of the middle cerebral artery. Stroke 32:2833-2835

85. Slotboom J, Kiefer C, Brekenfeld C et al (2004) Locally induced hypothermia for treatment of acute ischaemic stroke: a physical feasibility study. Neuroradiology 46:923-934

86. De Georgia MA, Krieger DW, Abou-Chebl A et al (2004) Cooling for acute ischemic brain damage (COOL AID): a feasibility trial of endovascular cooling. Neurology 63:312-317

87. Georgiadis D, Schwarz S, Kollmar R et al (2001) Endovascular cooling for moderate hypothermia in patients with acute stroke: first results of a novel approach. Stroke 32:2550-2553

88. Ding Y, Li J, Luan X et al (2004) Local saline infusion into ischemic territory induces regional brain cooling and neuroprotection in rats with transient middle cerebral artery occlusion. Neurosurgery 54:956-964

89. Patel MB, Kilgore KS, Ortolano GA et al (2001) Conditional blood reperfusion during angioplasty (CoBRA) treatment of acute myocardial infarction. Perfusion [Suppl] 16:39-49

90. Allen BS, Aeberhard E, Ignarro LJ (2003) Deep hypothermic circulatory arrest and global reperfusion injury: avoidance by making a pump prime reperfusate; a new concept. J Thorac Cardiovasc Surg 125:628-632

91. Langley SM, Chai PJ, Tsui SS et al (2000) The effects of a leukocyte-depleting filter on cerebral and renal recovery after deep hypothermic circulatory arrest. J Thorac Cardiovasc Surg 119:1262-1269

92. Allen BS, Castella M, Buckberg GD et al (2003) Conditioned blood reperfusion markedly enhances neurologic recovery after prolonged cerebral ischemia. J Thorac Cardiovasc Surg 126:1851-1858

93. Rimpilainen J, Pokela M, Kiviluoma K et al (2000) Leukocyte filtration improves brain protection after a prolonged period of hypothermic circulatory arrest: a study in a chronic porcine model. J Thorac Cardiovasc Surg 120:1131-1141 